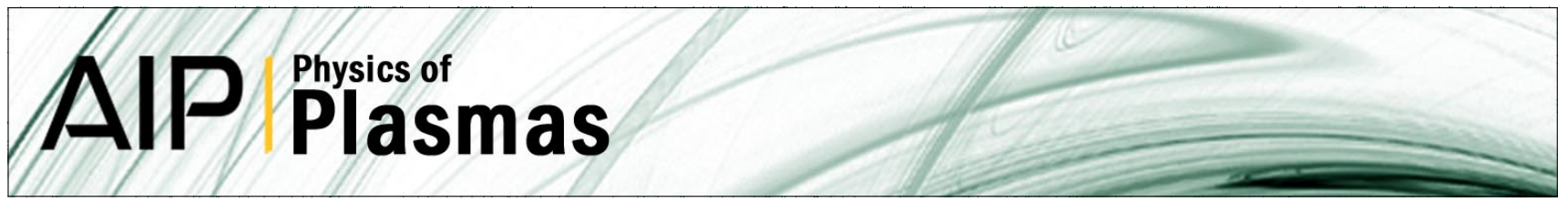

\title{
Examination of argon metastable atom velocity distribution function close to a conducting wall
}

N. Claire, S. Mazouffre, C. Rebont, and F. Doveil

Citation: Phys. Plasmas 19, 032108 (2012); doi: 10.1063/1.3692729

View online: http://dx.doi.org/10.1063/1.3692729

View Table of Contents: http://pop.aip.org/resource/1/PHPAEN/v19/i3

Published by the American Institute of Physics.

\section{Related Articles}

Production of internal transport barriers via self-generated mean flows in Alcator C-Mod

Phys. Plasmas 19, 056113 (2012)

Parallel heat transport in integrable and chaotic magnetic fields

Phys. Plasmas 19, 056112 (2012)

Experimental study of the ion current to a cylindrical Langmuir probe taking into account a finite ion temperature J. Appl. Phys. 111, 063303 (2012)

On the generation and disruption of a picosecond runaway electron beam during the breakdown of an atmospheric-pressure gas gap

Appl. Phys. Lett. 100, 134102 (2012)

First-order finite-Larmor-radius fluid modeling of tearing and relaxation in a plasma pinch

Phys. Plasmas 19, 055905 (2012)

\section{Additional information on Phys. Plasmas}

Journal Homepage: http://pop.aip.org/

Journal Information: http://pop.aip.org/about/about_the_journal

Top downloads: http://pop.aip.org/features/most_downloaded

Information for Authors: http://pop.aip.org/authors

\section{ADVERTISEMENT}

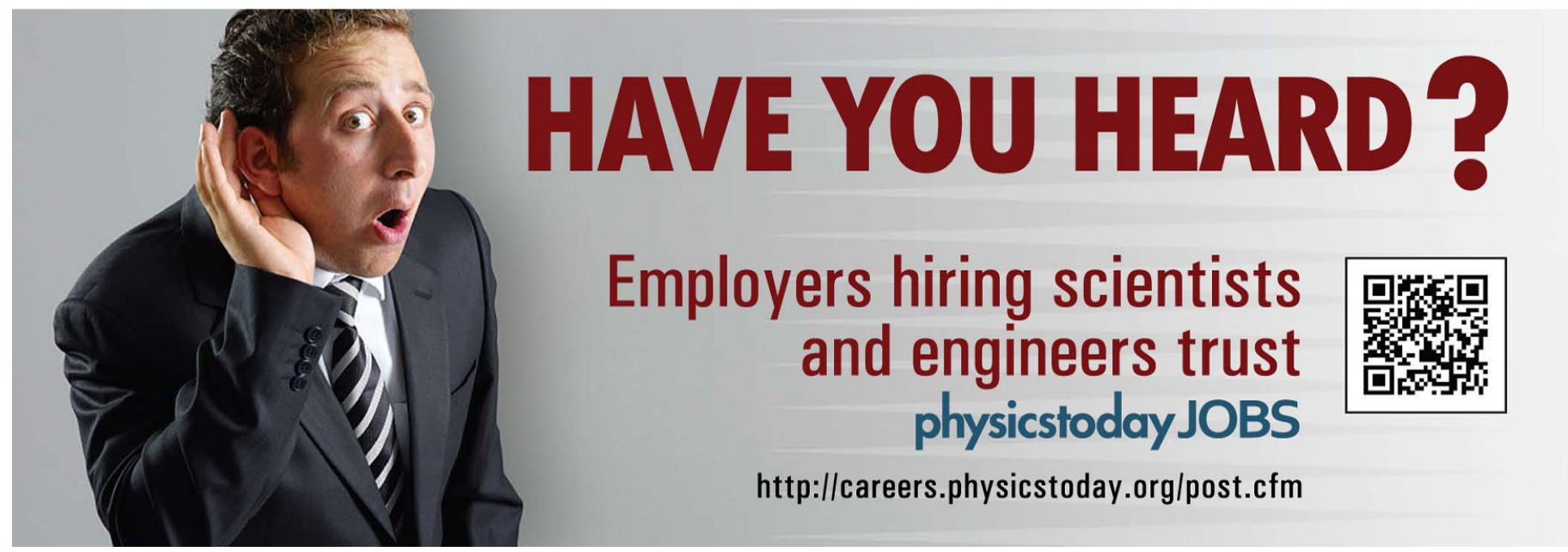




\title{
Examination of argon metastable atom velocity distribution function close to a conducting wall
}

\author{
N. Claire, ${ }^{1, a)}$ S. Mazouffre ${ }^{2}$ C. Rebont ${ }^{1}$ and F. Doveil ${ }^{1}$ \\ ${ }^{1}$ Equipe Turbulence Plasma, PIIM, CNRS/Université d'Aix-Marseille, Marseille, France \\ ${ }^{2}$ Laboratoire ICARE, CNRS, $1 C$ Av. de la Recherche Scientifique, 45071 Orléans, France
}

(Received 23 January 2012; accepted 17 February 2012; published online 14 March 2012)

The spatial evolution of the $1 s_{5}$ metastable argon atom velocity distribution function is recorded in the sheath and pre-sheath regions of a metallic wall using laser induced fluorescence (LIF) spectroscopy. Metastable argon atom temperature and fluid velocity are computed from measured data. Owing to the loss of metastable argon atom after a collision with the surface, the atom temperature seemingly decreases and the velocity increases when approaching the wall. These artifacts are carefully examined and explained in terms of changes in the metastable argon atom distribution function. In addition, the atom nonelastic reflection coefficient is computed from the ratio of outward to inward atom flux to the surface. This study indicates less than $1 \%$ of metastable atoms survive a collision with the metallic wall. (c) 2012 American Institute of Physics. [http://dx.doi.org/10.1063/1.3692729]

\section{INTRODUCTION}

Sheath and presheath studies are still an intense subject of research, because they are relevant in all plasma reactors. The evolution of metastable argon atoms population close to a conducting wall is relevant for plasma sputtering chemistry and deposition in industrial plasma reactor where the gas is not totally ionized and also in the edge of plasma fusion reactors where the plasma faces metallic surfaces. Since the work of Tonks and Langmuir ${ }^{1}$ the study of presheath and sheath potentials has motivated many analytical, ${ }^{2-8}$ and numerical ${ }^{9-18}$ works. A review article on the Bohm criterion and sheath formation can be found in Ref. 19 .

To experimentally obtain the metastable neutral argon velocity distribution functions (VDFs) in the vicinity of a metallic surface require a diagnostic having both a good spatial and velocity resolution. The laser induced fluorescence (LIF) diagnostic fulfills these requirements. Claire and co-workers ${ }^{20}$ have earlier obtained presheath and sheath ion velocity distribution functions in a multipolar argon discharge. Macko and Sadeghi ${ }^{21}$ have studied the behavior of the metastable argon atom VDF in the vicinity of a Pyrex surface.

In this contribution, the development of metastable $\mathrm{Ar}$ atom VDF is investigated as a function of the distance to a metallic wall. The profile of the Ar atom fluid velocity and temperature is computed from measured distributions. The apparent drop in temperature and rise in velocity is shown to be a direct consequence of interaction between metastable atoms and the wall. As we shall see, this work reveals the strong impact of walls on metastable species properties in a low-pressure environment, with direct consequences on the entire discharge dynamics. Since the effects of a metallic surface on temperature, velocity, and density of metastable neutral argon are significant, for low pressure up to about

\footnotetext{
${ }^{\text {a)} E l e c t r o n i c ~ m a i l: ~ n i c o l a s . c l a i r e @ u n i v-a m u . f r . ~}$
}

$20 \mathrm{~cm}$, these results are relevant for plasma and gas spectroscopy, for plasma assisted chemistry, deposition, surface treatments, and microelectronics, for example.

The paper is organized as follows: Sec. II is devoted to the description of the experimental apparatus. Section III presents a description of the LIF diagnostic. Section IV deals with the effect of laser absorption by Ar atoms on VDF. Section V shows the VDF measurements. Section VI presents the calculated nonelastic reflection coefficients. Conclusions are drawn in Sec. VII.

\section{EXPERIMENTAL APPARATUS}

Experiments are performed in a multipolar argon plasma device. ${ }^{20}$ A sketch of the device is displayed in Fig. 1. The $40 \mathrm{~cm}$ in diameter and $80 \mathrm{~cm}$ in length cylindrical plasma is ended by two conducting plates at floating potential. At about $8 \mathrm{~cm}$ from the device cylindrical wall, the plasma can be considered as unmagnetized. ${ }^{22}$ Note that no permanent magnet is installed on both ends. One end-plate has a $10 \mathrm{~mm}$ in diameter aperture to allow the laser beam to enter the vessel and propagate through the plasma. Electrons are emitted by a hot tungsten filament. The plasma is produced at lowpressure by electron bombardment on the seeding Ar gas.

In this study, the VDFs of argon atoms in a metastable state are probed in the vicinity of a $5 \mathrm{~cm}$ square metallic surface at floating potential $\phi_{p}=-0.95 \mathrm{~V}$ covered by a rugged layer diffusing the laser beam to avoid its specular reflexion. In the following, the negative coordinate $x$ represents distance from this surface located at $x=0$.

\section{LIF DIAGNOSTIC}

A complete description of laser induced fluorescence spectroscopy can be found in Ref. 23. In our case, argon atoms in the $1 s_{5}$ metastable state are optically pumped by exciting the $1 s_{5} \rightarrow 2 p_{9}$ transition at $\lambda=811.5311 \mathrm{~nm}$. Due to the Doppler shift, the laser frequency is resonant with the 


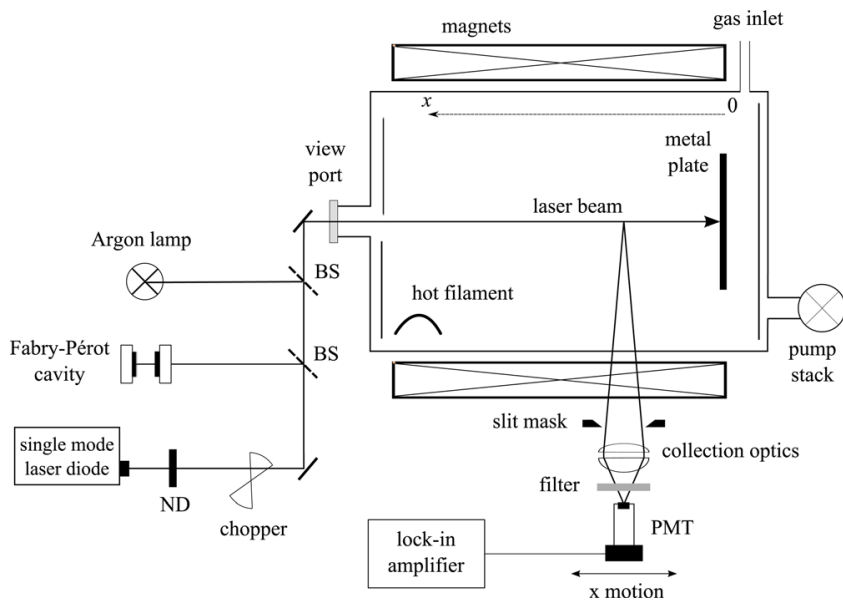

FIG. 1. Experimental setup: the multipolar device, the acquisition system, optics, and the laser beam in longitudinal position.

transition only if atoms have the velocity $v=c \frac{\nu_{l}-\nu_{0}}{\nu_{l}}$, where $\nu_{0}$ is the transition frequency for an atom at rest, $\nu_{l}$ is the laser frequency, and $c$ is the speed of light in vacuum. The subsequent fluorescence radiation is here collected at the excitation wavelength. The Ar atom VDF is obtained by scanning the laser frequency over the Doppler profile of the transition.

We use a single-mode tunable laser diode (TEC500 from Sacher) with a frequency width of $10 \mathrm{MHz}$ to pump atoms. The frequency changes are measured by a FabryPérot interferometer and the resonant frequency for atoms at rest by absorption in an argon lamp. The fluorescence is recorded by a photomultiplier tube throughout an objective with a narrow band filter of $1 \mathrm{~nm}$ and a slit inducing a spatial resolution of $0.1 \mathrm{~mm}$ along the laser beam but radially larger than the laser beam diameter $D=5 \mathrm{~mm}$. A classical phase sensitive technique is employed to improve the signal-tonoise ratio. The laser beam intensity is modulated at $1500 \mathrm{~Hz}$ by means of a mechanical chopper. The laser beam is shined perpendicular to the metallic surface as displayed in Fig. 1. Fluorescence light is recorded at $90^{\circ}$ with respect to the laser beam axis. A low power level of $200 \mu \mathrm{W}$ is used to avoid saturating the atomic transition. Only time-averaged VDF measurements are performed in this work.

\section{LASER BEAM ABSORPTION BY NEUTRALS}

Figure 2 shows the effect of the laser beam path length $L$ inside the plasma on $\operatorname{Ar}\left(1 s_{5}\right)$ VDF at argon gas pressure $P=2.510^{-3}$ mbar. The maximum of laser power absorption occurs when a maximum of atoms are in resonance with the laser frequency: in our case, it means when the velocity is close to zero. As the laser propagates through the plasma medium, a hole forms around $v=0 \mathrm{~m} / \mathrm{s}$, as can be seen in Fig. 2. Naturally, such a situation must be avoided as it prevents from measuring the VDF and from extracting atom flow properties.

The laser power absorption is governed by the BeerLambert's law. If $P$ is the laser power density for a path length $L$ in the plasma, $P_{0}$ is the laser power density ahead of the plasma, $n(v)$ is the number of atoms having velocity $v$ in

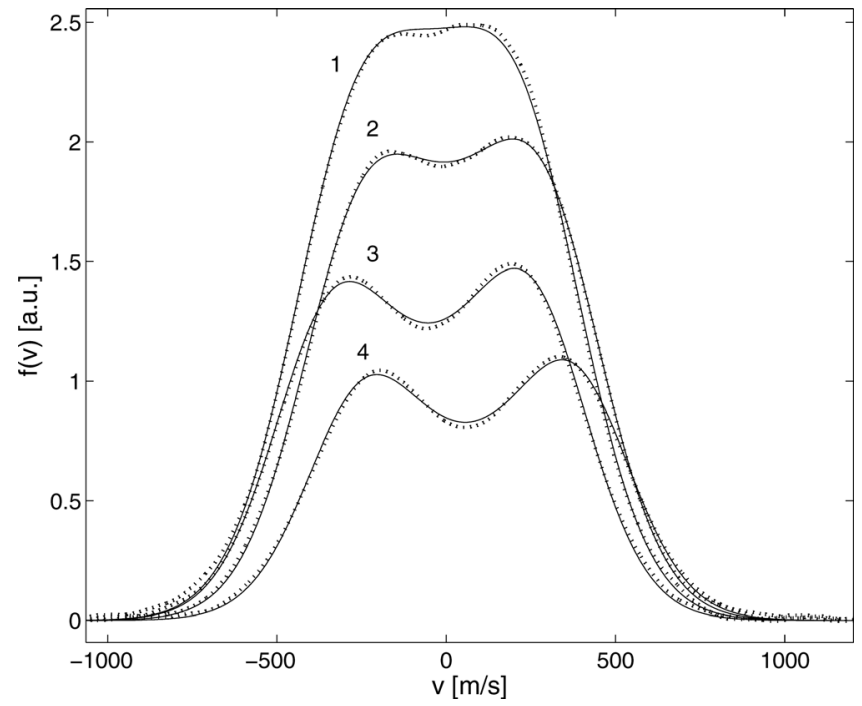

FIG. 2. Laser beam path length $\mathrm{L}$ effect in plasma on measured VDF (dashed curves) compared to theory (continuous curves) at $P=2.510^{-3}$ mbar. $L=(1) 30 \mathrm{~cm},(2) 33.6 \mathrm{~cm},(3) 42.5 \mathrm{~cm}$, and $(4) 54.5 \mathrm{~cm}$.

resonance with the laser frequency, and $\epsilon$ is the absorption coefficient

$$
P=P_{0} e^{-\epsilon n(v) L}
$$

For a thermalized plasma with a thermal velocity $v_{t h}$ and a drift velocity $v_{d}$

$$
n(v)=A e^{-\frac{1\left(v-v_{d}\right)^{2}}{2} v_{t h}^{2}}
$$

where $A$ is proportional to the total density. The laser induced fluorescence level $F(v)$ for a population of atoms with velocity $v$ is proportional to both $P$ and the numbers of atoms

$$
F(v)=\gamma \operatorname{Pn}(v)
$$

where $\gamma$ is a coefficient that depends principally on inelastic collisions and photon absorption cross-section.

Since, for the used laser diode, the laser power changes with the laser frequency, one can write down, $P_{0}=\alpha+\beta v$ after changing the laser frequency to atom velocity, thanks to Doppler effect, where $\alpha$ and $\beta$ are 2 constant coefficients specific to the laser diode. Therefore, the fluorescence level reads

$$
F(v)=\gamma(\alpha+\beta v) e^{-\epsilon L A e^{-\frac{1\left(v-v_{d}\right)^{2}}{2} v_{t h}^{2}}} A e^{-\frac{1\left(v-v_{d}\right)^{2}}{2 v_{t h}^{2}}} .
$$

Figure 2 shows simulations of the atomic line profile using Eq. (4). Note that the calculated profile is fitted to each measured one by a different set of coefficients and therefore these calculated profiles can only be qualitative. Nevertheless, the model shows that a precise description of laser power absorption effect by atom must be taken into account for high density neutral laser induced fluorescence measurements.

Fortunately, this effect can be avoided by reducing the atom density. In Fig. 3, measured line profiles are shown for 


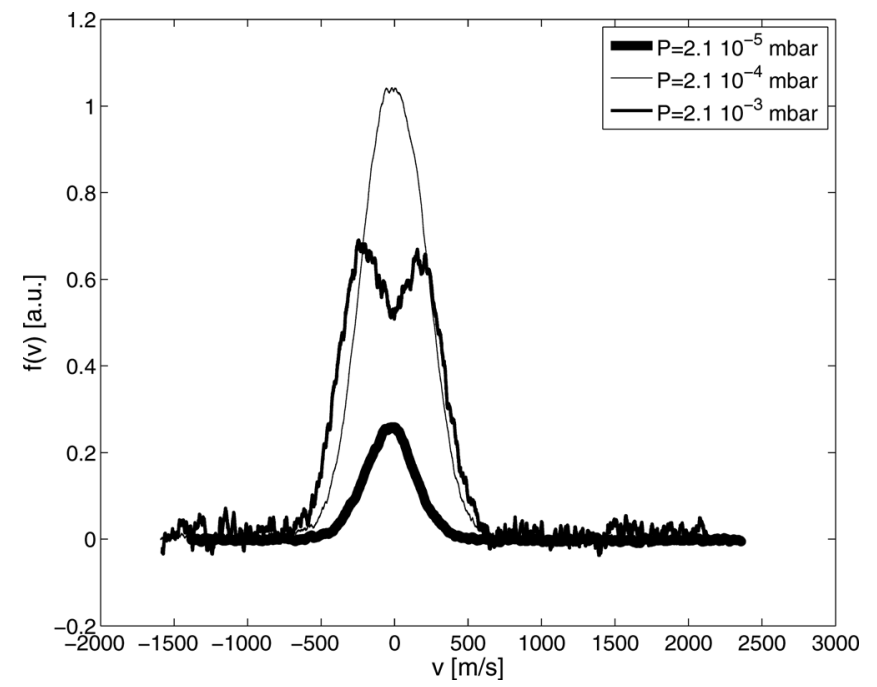

FIG. 3. Pressure effect on $\operatorname{Ar}\left(1 s_{5}\right) \mathrm{NVDF}$ for a fixed path length $L$.

various levels of the background pressure. At $P=210^{-5}$ mbar, no more pressure effect is seen. The following results are then obtained at this pressure.

\section{VDF IN THE VICINITY OF A SURFACE}

We present here measurements of $\operatorname{Ar}\left(1 s_{5}\right)$ VDF in the vicinity of a metallic surface at floating potential $\phi_{f}=$ $-0.95 \mathrm{~V}$. The experimental conditions are the following: $0.24 \mathrm{~A}$ of discharge current, $50 \mathrm{~V}$ of discharge voltage, and a background pressure of $2 \cdot 10^{-5}$ mbar.

The Ar fluid velocity and temperature are calculated from the first and second order moment of the measured VDF, respectively,

$$
\begin{gathered}
v_{f}=\frac{\int v f(v) d v}{\int f(v) d v}, \\
T=\frac{m}{k_{B}} \frac{\int\left(v-v_{f}\right)^{2} f(v) d v}{\int f(v) d v},
\end{gathered}
$$

where $m$ is the Ar atom mass and $k_{B}$ is the Boltzmann constant.

Figure 4 shows an example of $\operatorname{Ar}\left(1 s_{5}\right)$ VDF acquired at a distance $x=-219,-54,-14 \mathrm{~mm}$ from the surface. As can be seen, the VDF strongly departs from an equilibrium Gaussian distribution as the distance to the surface decreases. Owing to deexcitation of metastable argon atom after a collision with the surface, atoms with negative velocity component, e.g., velocity in the $-x$ direction, see Fig. 1, are no more detected. Indeed, in Fig. 4, the negative velocity part of the VDF is clearly truncated.

Therefore, the temperature calculated from LIF measurements seems to decrease with the distance to the surface, as shown in Fig. 5. T is slightly below the room temperature $\left(T_{\text {room }}=300 \mathrm{~K}\right)$ at the device center because of collisions with the device boundaries. The fluid velocity $v_{f}$ and the most probable velocity (peak of the VDF) $v_{\max }$ are plotted as a function of the distance to the surface in Fig. 6. The two

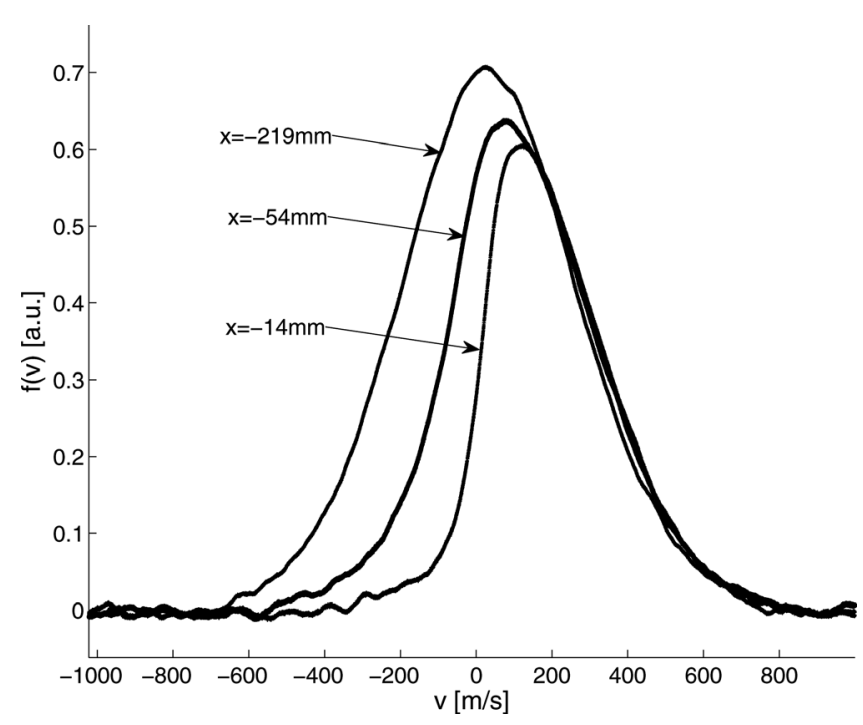

FIG. 4. Distribution function at $x=-219,-54,-14 \mathrm{~mm}$ from the surface.

velocities increase when moving towards the surface as a direct consequence of losses of metastable atoms with negative velocity component. Data in Fig. 6 also indicate that the gap between the two velocities increases when approaching $x=0$.

Losses of metastable argon atoms on the surface cannot explain the on-axis distribution of $v_{\max }$. As only atoms with negative velocity are lost in an inelastic collision with the surface, the most probable velocity should remain close to zero. A possible explanation for the change in $v_{\max }$ is diffusion in velocity phase space due to collisions with particles from the residual gas.

As can be observed in Figs. 5 and 6, data are scattered very close to the surface. In Fig. 6, the most probable velocity even seems to decrease. This tendency is certainly due to laser beam diffusion onto the surface. A small fraction of the beam is reflected back and a twin VDF, yet attenuated, is detected with an opposite Doppler shift.

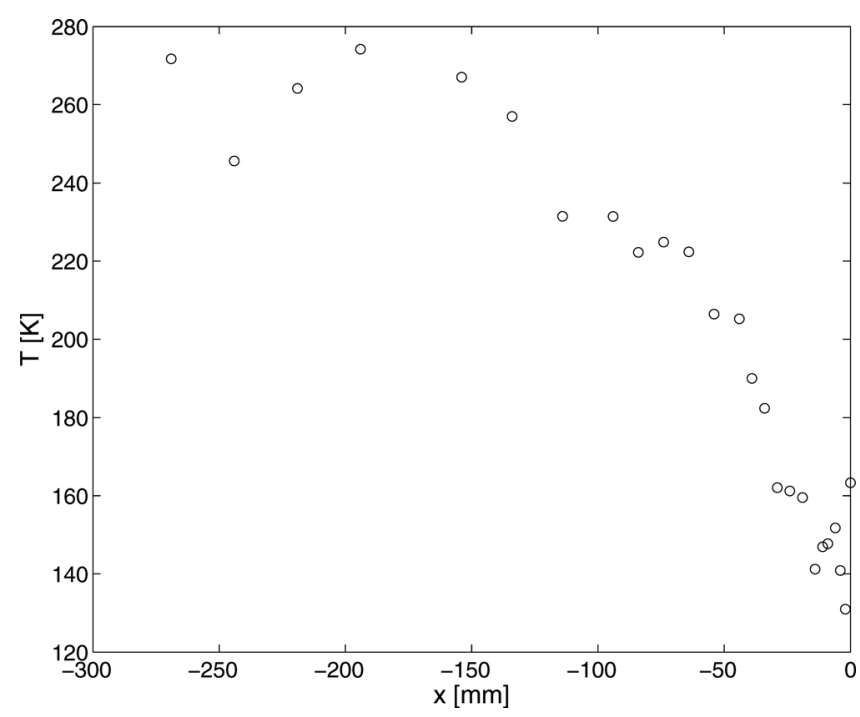

FIG. 5. Temperature $T$ with respect to the distance $x$ to the surface. 


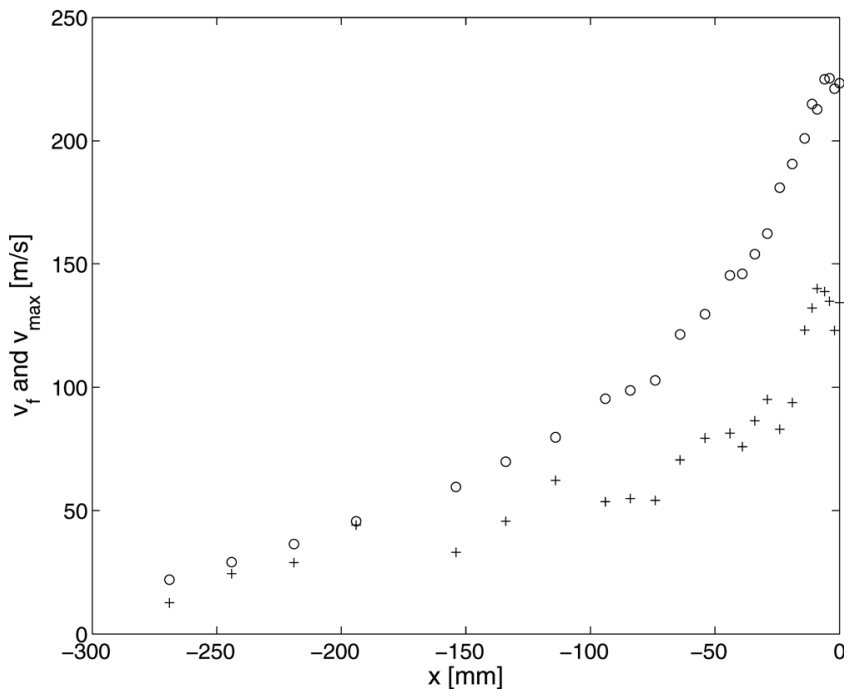

FIG. 6. Fluid velocity $v_{f}$ (circles) and most probable velocity $v_{\max }$ (crosses) of the atom VDF against the distance $x$ to the surface.

\section{NONELASTIC REFLECTION COEFFICIENT}

The nonelastic reflection coefficient $R$ for $1 s^{5}$ argon atoms on a metallic surface can be computed as follows: ${ }^{21}$

$$
R=\frac{\left|J^{-}\right|}{\left|J^{+}\right|}
$$

where

$$
J^{+}=n(v>0) \int_{0}^{\infty}|v| f(v) d v
$$

and

$$
J^{-}=n(v<0) \int_{-\infty}^{0}|v| f(v) d v
$$

are the atom flux directed towards the surface and the flux in the opposite direction, respectively. Because of the measured drift of $v_{\max }$, another nonelastic reflection coefficient can be computed, similar to $R$, but fluxes are then computed in the reference frame with velocity $v_{\max }$

$$
R^{*}=\frac{n\left(v<v_{\max }\right) \int_{-\infty}^{v_{\max }}|v| f(v) d v}{n\left(v>v_{\max }\right) \int_{v_{\max }}^{\infty}|v| f(v) d v}
$$

The development of $R$ and $R^{*}$ along the axis is displayed in Fig. 7. For $x>0$, the two coefficients image somehow the departure from the equilibrium distribution. As can be seen, even $25 \mathrm{~cm}$ away from the metallic wall $R$ and $R^{*}$ are not equal to 1 , which clearly demonstrates surfaces have a drastic impact upon excited particle energy distribution in a lowpressure environment. The value computed when $x$ tends to zero corresponds to the reflection coefficient of $1 s^{5} \mathrm{Ar}$ atoms on the metal surface. One finds a value below $1 \%$ for $R$ meaning that only a few metastable atoms survive a wall-collision. For comparison, but in different plasma conditions, RF ionization, ${ }^{3} P 2$ metastable argon level, $P=9.10^{-4}$ mbar, Macko and Sadeghi ${ }^{21}$ have obtained $R=0.25$ near a Pyrex surface.

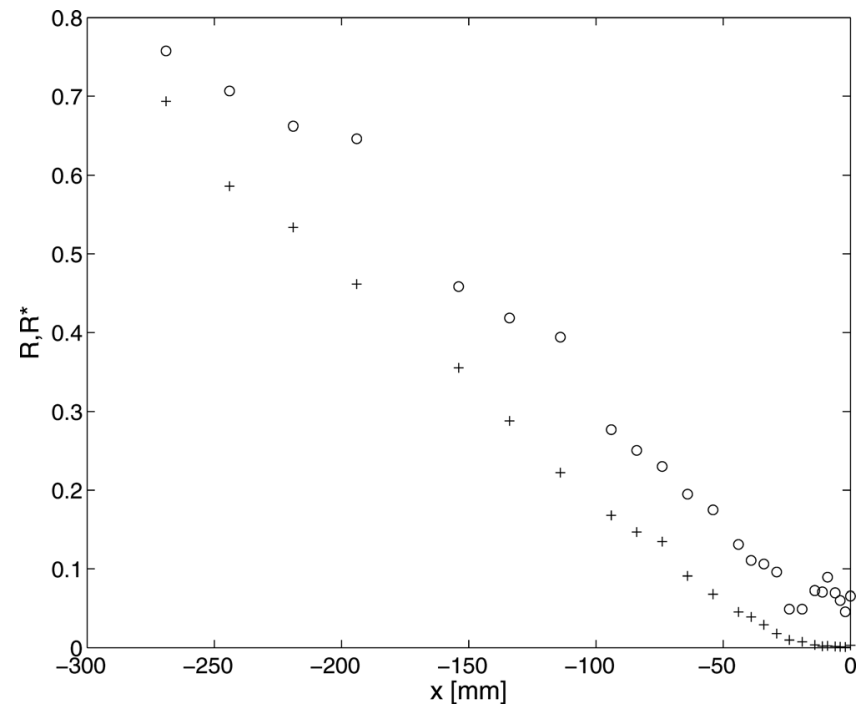

FIG. 7. Reflected coefficient $R$ (crosses) and $R^{*}$ (circles) with respect to the distance $x$ to the surface.

\section{CONCLUSION}

Velocity distribution function measurements nearby a metallic surface demonstrate that LIF diagnostic is a powerful nonintrusive diagnostic which allows to easily compute temperature, fluid velocity, and nonelastic reflected coefficients. Nevertheless, our results show that low pressure and low laser density power must be used to avoid artifacts like saturation effect and laser power absorption by atoms. Losses by nonelastic collisions of $\operatorname{Ar} 1 s^{5}$ metastable atoms on a metallic surface occur and involve changes in VDF shape: a fluid velocity drift and a temperature cooling with respect to the distance to the surface. Because of the unexpected acceleration of the most probable velocity, velocity diffusion, or specific argon atom metastable collisions are likely to play a role. Therefore, two nonelastic coefficients are calculated with respect to the distance to the surface using outward and inward neutral flux in the laboratory frame or outward and inward flux shifted by $v_{\max }$, the velocity at the VDF maximum. Close to the surface, less than $1 \%$ of metastable atoms survive a collision with the metallic wall because all metastable atoms with negative velocity have made a nonelastic collision with the surface and can no more be detected.

Contrary to the well-admitted picture, this study indicates a metal wall is not a perfect sink for excited species. Although the fraction of excited species, here in the form of metastable Ar atoms, which survive a collision with the surface is rather low, it certainly influences the discharge dynamics and parameters. As a consequence, recombination of excited atoms and molecules at walls must be treated with care in processes that rely on reactive plasmas like thin film deposition and etching of microstructures. Not only the macroscopic properties of the plasmas depend on plasma-walls interactions but also the shape of the velocity distribution function for excited atoms. This is a critical point in the fields of atomic physics and spectroscopy, wherein lowpressure discharges are often used for measurement of fundamental quantities. 


\section{ACKNOWLEDGMENTS}

This work was supported by Réseau des Plasma Froids of the CNRS. Cyril Rebont benefited from a grant by Ministère de la Recherche.

${ }^{1}$ L. Tonks and I. Langmuir, Phys. Rev. 34, 876 (1929).

${ }^{2}$ E. R. Harrison and W. B. Thompson, Proc. Phys. Soc. London 74, 145 (1959).

${ }^{3}$ L. S. Hall, Proc. Phys. Soc. London 80, 309 (1962).

${ }^{4}$ A. Caruso and A. Cavaliere, Nuovo Cimento 26, 1389 (1962).

${ }^{5}$ G. A. Emmert, R. M. Wieland, A. T. Mense, and J. Davidson, Phys. Fluids 23, 903 (1980).

${ }^{6}$ S. D. Baalrud, J. D. Callen, and C. C. Hegna, Phys. Rev. Lett. 102, 245005 (2009).

${ }^{7}$ W. J. Miloch, N. Gulbrandsen, L. N. Mishra, and Å. Fredriksen, Phys. Plasmas 18, 083502 (2011).

${ }^{8}$ R. C. Bissel and P. C. Johnson, Phys. Fluids 30, 2264 (1987).

${ }^{9}$ S. A. Self, Phys. Fluids 6, 1762 (1963).
${ }^{10}$ R. J. Procassini, C. K. Birdsall, and E. C. Morse, Phys. Fluids B 2, 3191 (1990).

${ }^{11}$ L. A. Schwager and C. K. Birdsall, Phys. Fluids B 2, 1057 (1990).

${ }^{12}$ T. E. Sheridan and J. Goree, Phys. Fluids B 3, 2796 (1991).

${ }^{13}$ J. M. Grossman, S. B. Swanekamp, and P. F. Ottinger, Phys. Fluids B 4, 44 (1992).

${ }^{14}$ L. Shott, Phys. Fluids 30, 1795 (1987).

${ }^{15}$ K. Sato and F. Miyawaki, Phys. Fluids B 4, 1247 (1992).

${ }^{16}$ T. E. Sheridan, N. St. J. Braithwaite, and R. W. Boswell, Phys. Plasmas 6, 4375 (1999).

${ }^{17}$ T. E. Sheridan, Phys. Plasmas 8, 4240 (2001).

${ }^{18} \mathrm{~F}$. Valsaque, ${ }^{\circ} \mathrm{Ph}$.D. dissertation, University of Nancy I, France, 2002.

${ }^{19}$ K. U. Rieman, J. Phys. D: Appl. Phys. 24, 493 (1991).

${ }^{20}$ N. Claire, G. Bachet, U. Stroth, and F. Doveil, Phys. Plasmas 13, 062103 (2006).

${ }^{21}$ P. Macko and N. Sadeghi, Plasma Sources Sci. Technol. 13, 303 (2004).

${ }^{22}$ R. J. Taylor, K. R. MacKenzie, and H. Ikezi, Rev. Sci. Instrum. 44, 726 (1978).

${ }^{23}$ D. N. Hill, S. Fornaca, and M. G. Wickham, Rev. Sci. Instrum. 54, 309 (1983). 